\title{
Biological Photonic Crystals - Revealed by Multi-photon Nonlinear Microscopy
}

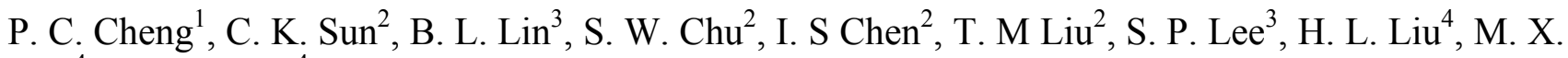
$\mathrm{Kuo}^{4}$ and D. J. Lin ${ }^{4}$

${ }^{1}$ Department of Electrical Engineering, State University of New York, Buffalo, NY 14260 USA

${ }^{2}$ Department of Electrical Eng., Natl. Taiwan Univ., Taipei, Taiwan, 10617, Republic of China

${ }^{3}$ Molecular \& Cell Biology Div., Development Ctr for Biotechnology, Taipei, Taiwan, 10659, ROC.

${ }^{4}$ Department of Physics, Natl. Taiwan Normal University, Taipei, Taiwan, 116, Republic of China

Highly optically active nonlinear bio-photonic crystal structures in living cells are studied by a novel multi-modal nonlinear microscopy. Numerous biological structures, including stacked membranes and arranged protein structures are highly organized in nano scale and are found to exhibit strong optical activities through second-harmonic-generation (SHG) interactions, behaving similar to manmade nonlinear photonic crystals. The microscopic technology used in this study is based on a combination of different imaging modalities including SHG, third-harmonic-generation (THG), and multi-photon-induced fluorescence. With no energy deposition during harmonic generation processes, the demonstrated nonlinear-photonic-crystal-like SHG activity in highly organized biological nano-structrues is useful for investigating the dynamics of structure-function relationship at subcellular level and is ideal for studying living cells. In order to allow both SHG and THG fall within the visible spectrum, and also achieve low illumination attenuation $[1,2,3,4]$, we move the excitation wavelength to the 1200-1350nm regime by using a Cr:forsterite laser with center wavelength around $1230 \mathrm{~nm}$, resulting not only the visible but also the near infrared (NIR) spectrum open for simultaneous recording of 2PF, three-photon fluorescence (3PF), SHG and THG. Our previous studies using $1230 \mathrm{~nm}$ light indicated efficient excitation of $2 \mathrm{PF}$ and $3 \mathrm{PF}$ in common bioprobes $[3,5]$. Figure 4 shows the experimental setup of a multi-modal nonlinear laser scanning microscope. (Figure 4: Laser: Cr:forsterite femtosecond laser centered at 1230nm; BS: beam splitter; L: mercury lamp; S: sample; TS: 3-dimensional translation stage; F: color filter; SM: spectrometer; C: computer, CA: camera).

Figure 1 is a set of $x-y-\lambda$ images of the adaxial surface of rice leaf showing dumb-bell-shaped silica cells ( $\mathrm{s}$, profiled by dashed line). The illumination polarization is shown as white arrows in each image. The orientation of SHG images obviously follows the illumination polarization while no correlation can be found in THG images. Scale bar $=15 \mu \mathrm{m}$. Figure 2 shows a set of $\mathrm{x}-\mathrm{y}-\lambda$ images of a live mesophyll cell from Commelina communis L. revealing the distribution of chloroplasts as imaged by THG, SHG and 2-photon fluorescent modes. The enlarged images show the individual chloroplasts (Fig. 3). By comparing the SHG image with TEM images of similar samples, the crescent-shaped SHG image was identified to be the grana and the oval-shaped structures are starch granules. (Scale bar: $15 \mu \mathrm{m}$ ). Figure 5 demonstrates the starch granules in a piece of fresh potato can generate strong frequency doubled output. An objective lens was used to focus the $1230 \mathrm{~nm}$ illumination onto a small piece of potato. Our results reveal photonic crystal-like optical activity in living cells using a multi-modal microscopy. Highly organized nano-period structures in biological samples exhibit strong SHG activities resembling nonlinear photonic crystals and thus can be treated as "nonlinear bio-photonic crystals". Numbers of biological structures exhibit strong bio-photonic effect, such as cell wall, starch granule, silica deposition, cuticular papillae on the epidermal cell of rice leaf, crystalline myosin and actin nano-filaments in the myofibrils of skeletal muscles, and grana in chloroplast. 
[1] Lin, B. L. et al., (2001): Maize Genetics Cooperation News Letters 75, 61-62.

[2] Cheng, P. C. et al., (1998): J. Microsc. 189:199-212.

[3] Cheng, P. C. et al., (2000): Scanning, 22, 187-188.

[4] Cheng, P. C. et al., (2001): SPIE Proceedings, 4262, 98-103.

[5] Liu T. M. et al., (2001): Scanning 23, 249-254.

Supported by grants from the National Science Council of ROC [NSC89-2215 -E-002-064, NSC892112-M-002-082 (CKS)], NSC89-2811-E-002- 0058 (PCC). [NSC89-2311-B-001-134, NSC892311-B-001-137 (BLL)], Academia Sinica (BLL). National Taiwan University (CKS).

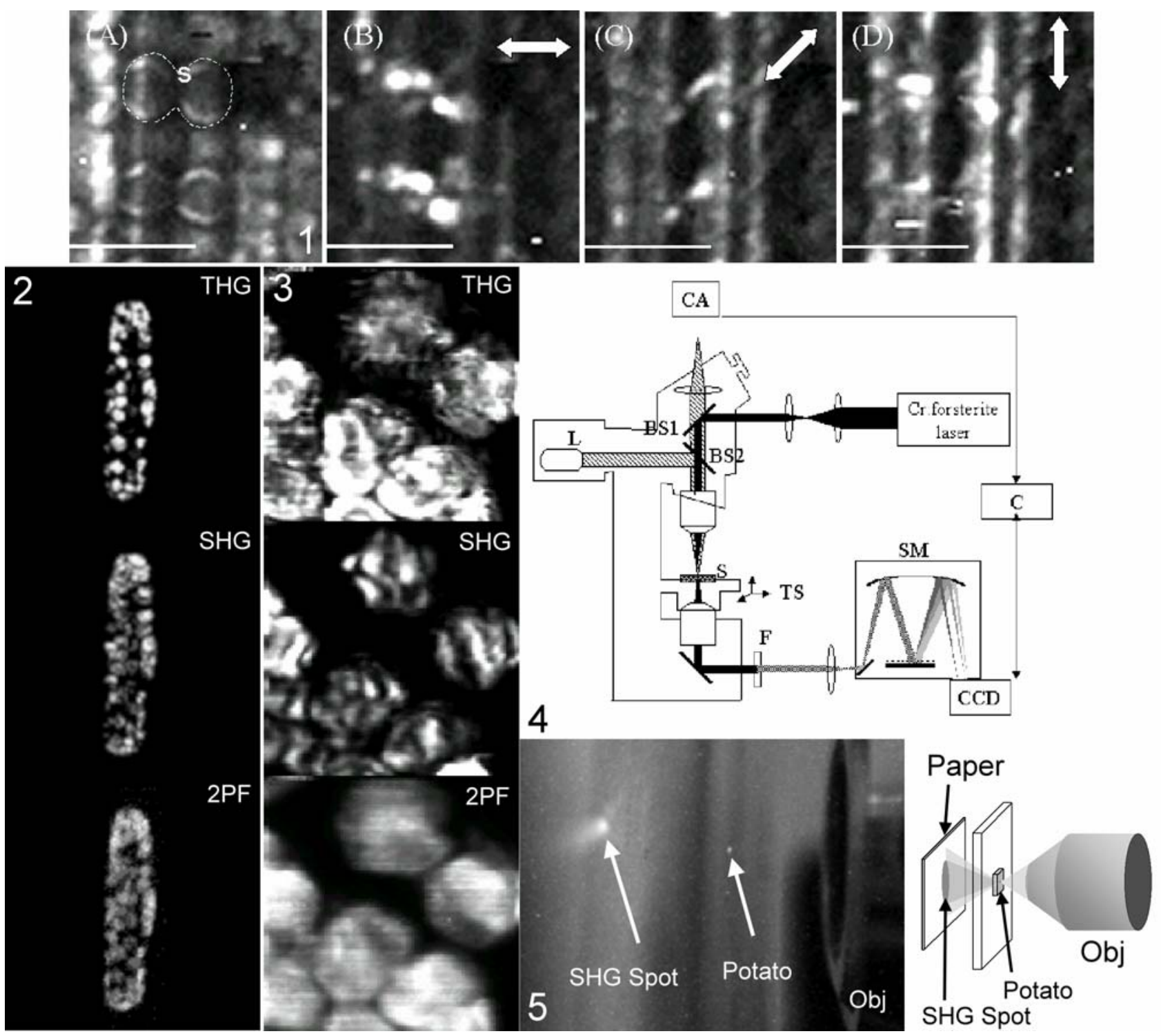

FIG 1A: Silica cell (outlined by dotted lines) on the surface of rice leaf. 1B-1D: SHG images at varying polarization planes (white double-head arrows). Scale bar $=15 \mu \mathrm{m}$.

FIG 2: Live mesophyll cell of C. communis imaged by THG, SHG and autofluorescent modes.

FIG 3: Chloroplasts in live mesophyll cell of $C$. communis imaged by THG, SHG and autofluorescent. FIG 4: Experimental setup. L: light source; BS1, BS2: beam splitter; S: sample; SM: spectrometer. FIG 5: strong frequency doubled output generated by a piece of fresh potato. An objective lens was used to focus the $1230 \mathrm{~nm}$ illumination onto a small piece of potato as shown in the diagram. 